\title{
Functional Relation of Pacinian Corpuscle to Vascular System
}

\author{
Eizo Yamashita* and Nicholus Buendia $\uparrow$ \\ Department of Physiology (Prof. W. R. Loewenstein), \\ College of Physicians and Surgeons, Columbia University, New York
}

\begin{abstract}
Afferent impulses coming from the Pacinian corpuscles in response to mesenteric arterial pulsation were observed at the peripheral cut end of the cat's mesenteric nerves. The correlation between blood pressure and impulse frequency was not always fixed. There seemed to be differences in the behavior of the corpuscles. In some of them, the impulse frequency was increased by a rise in blood pressure, whereas in others it was depressed by blood pressure elevation. The result was explained from the observation that the mesenteric arteries were dislocated in such a way with their increased constriction that they drew toward or withdrew from the Pacinian corpuscles. Possible reflex for controlling abdominal functions was discussed with relation to vascular change.
\end{abstract}

Since recent electro-physiological methods were introduced into the study on sense organ, the property of isolated Pacinian corpuscles has been much clarified by many workers. ${ }^{1-8}$ But their true role in animal and human life has remained still to be clarified. In the cat, many Pacinian corpuscles are situated in the mesentery and connective tissue surrounding the pancreas. It is not probable to regard them as a receptor subserving simply touch sensation of abdominal organs, and other anatomists ${ }^{9}$ expected that they might contribute to circulatory control. In the cat's mesentery, in fact the Pacinian corpuscles lie in great abundance along the arteries running radially from the mesenterial root. So it is likely that they might be stimulated by the systolic arterial thrusts.

Gammon and Bronk ${ }^{10}$ approached the problem by observing the relation between impulse frequency from the Pacinian corpuscles and variation of blood pressure in the cat. They observed that injection of fluid into the vessel produced an increased dicharge from the corpuscles in association with a rise in blood pressure, and hemorrhage did opposite results. They noticed, however, a marked decrease of the impulse discharge with increase in blood pressure produced by adrenaline, and increased discharge with decrease in the pressure by acetylcholine. In explanation for this opposite manner of Pacinian corpuscles, they assumed that the sensory discharge of the Pacinian corpuscles is not directly related to the level

Received for publication, May 16, 1968.

* Present address: Miyagi Teachers College, Sendai, Japan.

$\uparrow$ Present address: Morristown Memorial Hospital. Morristown, N.J., U.S.A. 
of general blood pressure, but rather to the degree of distention of the local blood vessels.

In the present paper, the characters and response patterns of the blood vessel and the corpuscle in a living cat's mesentery were studied with changing blood pressure. The obtained results gave some new suggestions to the explanation of the problems.

\section{METHODS}

A cat was fixed in a supine position after anesthetized with an intra-peritoneal injection of Nembutal $(3.5 \mathrm{mg} / \mathrm{kg})$, the abdomen was opened along the median line and kept widely open by specially devised retractors. Fig. 1 illustrates an arrangement of the preparation. A part of the intestine (I) with the mesentery containing Pacinian corpuscles was mounted on a lucite plate $(\mathrm{P})$ which was hung inside the opened abdominal cavity. The cat was warmed so as to keep normal body temperature, and warmed water at about $40^{\circ} \mathrm{C}$ was perfused round inside of the plate by an electric pump and warmed Krebs solution was often sprayed on the mesentery to prevent it from drying and cooling. A heparin coated polyethylene tube (C) was inserted into the braneh of a mesenterie artery, and the other end was connected to a strain-gauge for recording the local blood pressure. Sometimes piezo-electric crystal (CR) was used to record the amplitude of pulsation. A glass stylus $(G)$ cemented on the crystal transmitted the pulsatory movements of the artery to the crystal, and piezo-electric change amplified was used as an indication of the amplitude of the pulsation. The nerve trunk (N) running along the same artery was separated from surrounding tissue and was cut. After removing the connective tissue and sheath, the distal end of the nerve was picked up with the tips of two platinum electrodes (E), and mineral oil was put into the abdominal cavity to dip the lifted nerve trunk. This trunk contains the axons coming from several Pacinian corpuscles located close to the artery.

In most cases blood pressure was changed temporarily to various levels by an intravenous injection of $2-3 \mathrm{ml}$ of $0.00005 \%$ noradrenaline solution or with hemorrhage. In a few cases an intravenous injection of Krebs solution or acetylcholine solution was adopted to compare the effect of blood volume change with that of the reagents.

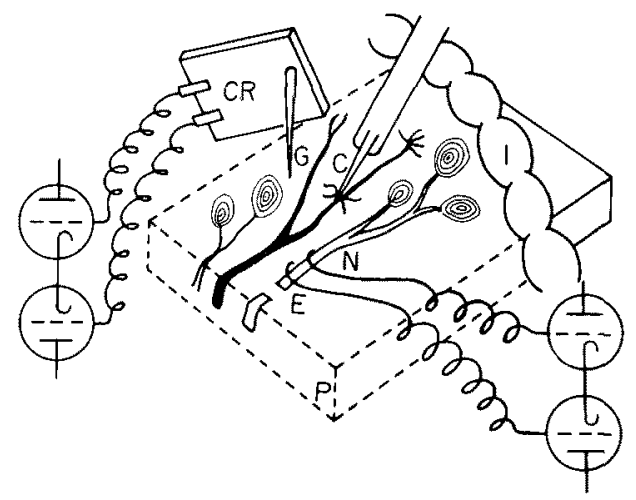

Fig. 1. Arrangement of the preparation and experimental set-up. Explanation in text.

\section{Results}

From the peripheral cut end of isolated mesenteric nerve trunk, spontaneous unit discharges were frequently obtained. When slight mechanical vibrations 
were given to the mesentery, the same unit increased its firing rate markedly, and discharges from different units were also frequently observed simultaneously. Units without firing on mechanical stimulations were all excluded because they did not seem to come from the Pacinian corpuscles.

Most of the spontaneous unit discharges showed frequently such a feature that a single spike or burst of 3-4 spikes appeared synchronously with every systolic phase of pulsation. In this case the impulse ceased to appear after occlusion of artery running along the nerve with a glass rod, and this gives a satisfactory evidence that the Pacinian corpuscles were excited in response to every arterial pulsation. On the other hand, such spontaneous discharges with fairly high frequency were sometimes obtained, which responded to mechanical stimulus but had no definite relation with pulsation at any blood pressure levels. The possibility that other types of endings take part in mechanical information was not entirely eliminated.

\section{Discharge patterns in response to blood pressure change}

Fig. 2 shows an example of unit discharge in which the frequency of impulse discharge accompanied a rise in blood pressure level. On the other hand, however, impulses became depressed or stopped as blood pressure rose, and they much increased as blood pressure fell again as shown in the example of Fig. 3 . In this case the stylus lightly attached to the artery gave the evidence that the amplitude of pulsatory movement still increased with rising blood pressure, and decreased with falling blood pressure. Gammon and Bronk ${ }^{10}$ presumed that the discharge character was related to the degree of distention of the local vessels, but not to the level of mean blood pressure. In the present experiments, however, noradrenaline brought about both response patterns. The following observations

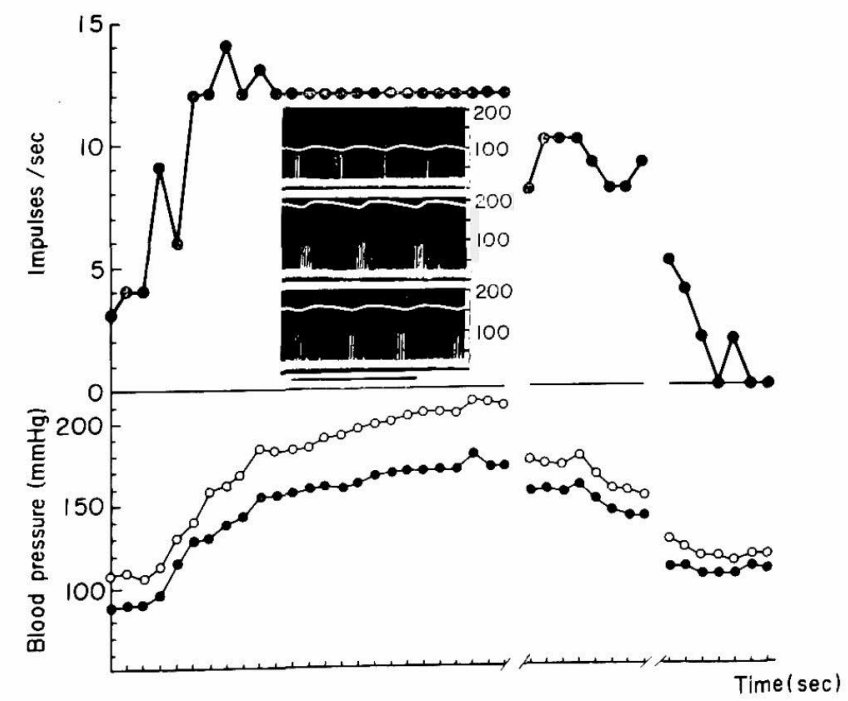

Fig. 2. Correlation between impulse number and blood pressure level. 


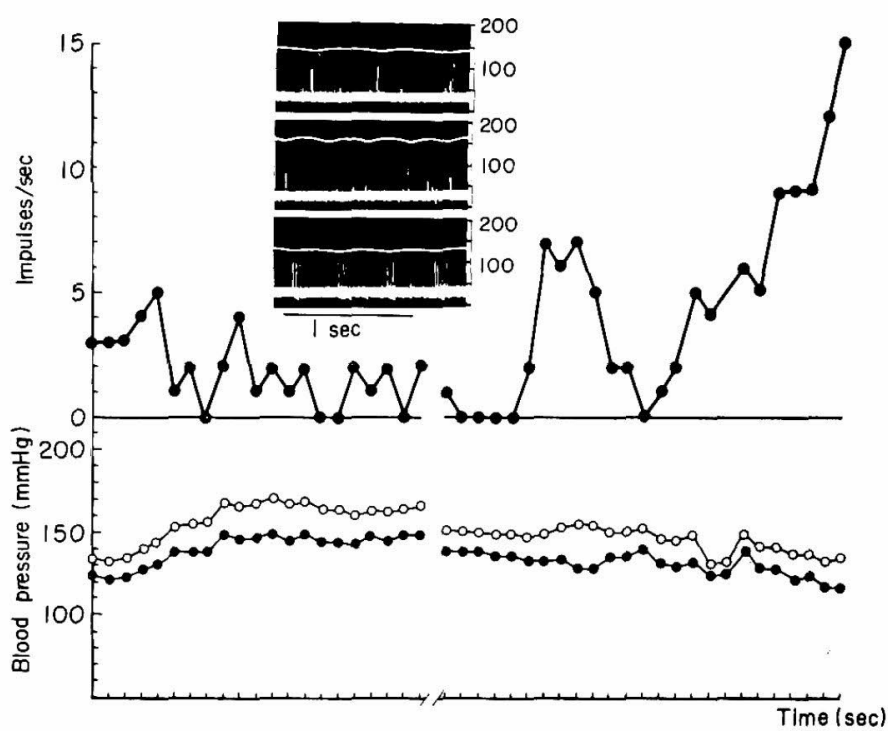

Fig. 3. Correlation between impulse number and blood pressure level.

will be enough to give an explanation for the process producing two opposite response patterns.

\section{Dislocation of artery and its stimulus influence upon the Pacinian corpuscles}

The tip of stylus located successively at several different points of the mesentery close to the artery was sensitive enough to pick up slight mechanical movement caused by pulsation. Fig. 4 shows a series of simultaneous records of mechanical movement and blood pressure level. The tip positions of stylus in relation to the artery are indicated in the right diagram of Fig. 4 . The mechanical movement picked up at the point $\mathrm{A}$ increased with rising blood pressure, but at

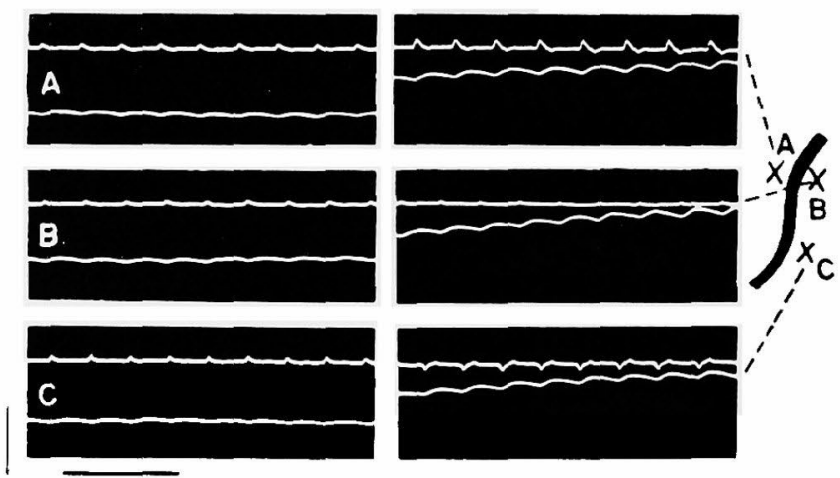

Fig. 4. Right column concerns amplitude of pulsation (each upper beam), left column concerns the control. The series A indicates pulsatory movement recorded at the site of $\mathrm{A}$ as marked in the diagram, and the $\mathrm{B}$ and $\mathrm{C}$ series are those recorded at the site of $\mathrm{B}$ and $\mathrm{C}$, respectively. Horizontal bar indicates one second, 
the opposite side across the artery - at the point B - the movement decreased under high blood pressure level. And at another point marked with $\mathrm{C}$, the polarity of pulsatory movement was reversed as the pressure rose. With microscopic observation the artery was seen to be dislocated gradually towards the left side-point A side - when blood pressure was rising. So it is proper to consider that the tip of stylus attached to the mesentery at the point $A$ was approached by the artery and received stronger pulsatory effect with rising blood pressure. On the contrary, on the opposite side the artery went away as the pressure was rising and only weaker effect was given to the stylus located at the point $B$. If the stylus is assumed to be replaced by a Pacinian corpuscle, the same process will be expected. Fig. 5 shows an example of the simultaneous records of mechanical movement and unit discharge. The stylus was attached onto the mesenteric tissue close to the corpuscle located along an arterial branch. The series A, in which records were obtained when the mesentery was stretched on a plate so as to exert some tension, illustrates that a Pacinian corpuscle responded to pulsation at high pressure level. But after the mesentery was much relaxed the polarity of movement was reversed and impulses stopped, as shown in series $B$. The change of tension seems to alter the positional situation between the corpuscle and artery. But in this case, the artery was observed to dislocate towards only the same side regardless of any positional situations. So it seems to be difficult to reverse one response pattern experimentally to the other on the same unit.
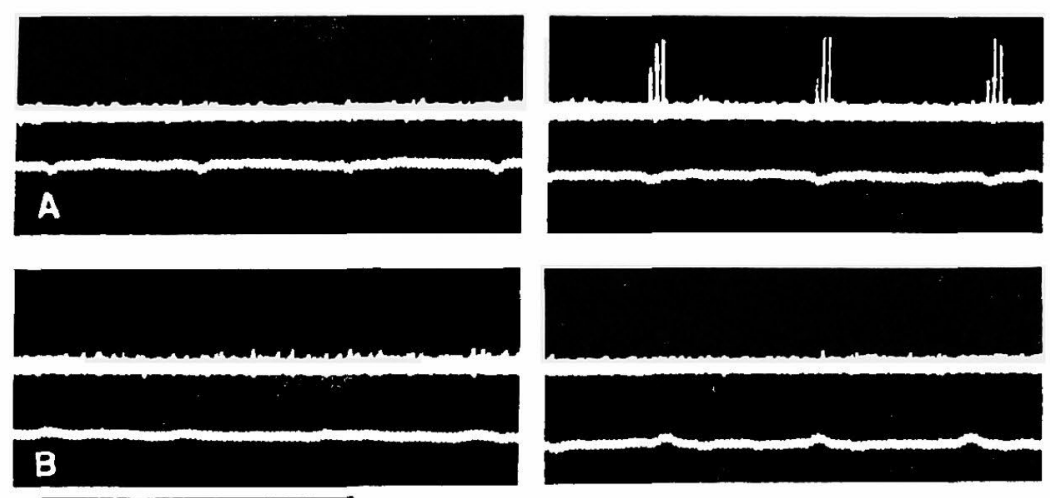

Fig. 5. Simultaneous records of pulsatory amplitude and unit discharge. $A$ and $B$ series indicate the records obtained from the stretched mesentery and those from the same but relaxed mesentery respectively.

\section{Possible vasomotor reflex on Pacinian corpuscle}

Judging from the result that Pacinian corpuscles catch some information about the vascular state, it is probable to expect a feedback system to control the blood pressure by means of the impulse rate coming from the Pacinian corpuscles. With attention focussed on the possible reflex, local and systemic blood pressures were 
recorded many times from the mesenteric artery or common carotid artery under a sustained application of intermittent vibrations with the same rhythms as those of the pulsation or with various higher rhythms. The applied vibration brought about burst of impulses from many corpuscles and probably sustained them in an over-accelerated state. But the blood pressure always showed no detectable change, and the results were the same in cats anesthetized with chloralose. On the other hand, when a part of the mesentery containing arterial branches was lightly tapped by the fingers, both the systemic and mesenteric blood pressure decreased temporarily by $10-15 \mathrm{~mm} \mathrm{Hg}$ and came back to the original level within approximate 10 seconds, even when the tap was continued. But this had no relation with the function of Pacinian corpuscles.

The taps on the region including the Pacinian corpuscles in abundance but few arterial branches bring about no effect on the blood pressure as the white arrow shows in Fig. 6, but the black arrow shows that the taps on arterial branches cause a temporary fall of blood pressure, even if no corpuscles are included in the region.

Since the similar temporary blood pressure fall is observed with the taps of arteries other than mesentery, such blood pressure drop seems to be a result of vasomotor reflex due to the mechanical stimulation of arteries.

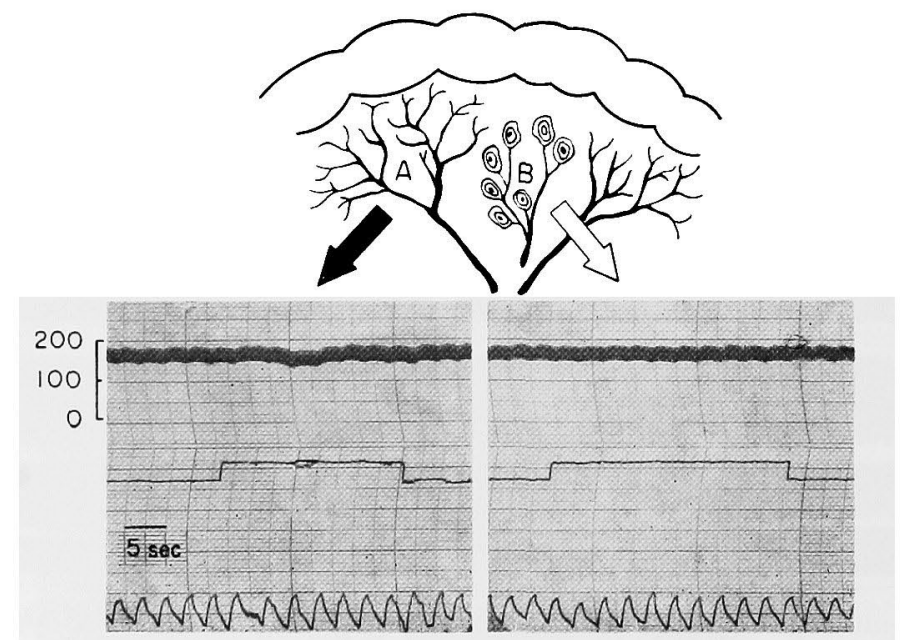

Fig. 6. Upper record indicates blood pressure in $\mathrm{mm} \mathrm{Hg}$, and middle and lower records tapping duration and respiratory curve respectively. Explanation in text.

\section{Discussion}

In order to account for the reason why the Pacinian corpuscle responds in a manner opposite to an increase of blood pressure caused by vasoconstriction and one due to augmented blood volume, Gammon and Bronk ${ }^{10}$ presumed that the intrinsic vessels of Pacinian corpuscles are responsible for the excitation of the 
corpuscles. But by way of recent electron-microscopic observations the vessels reaching the nerve ending in Pacinian corpuscles have not been approvable.

The present experiments in this paper show that the response manner of Pacinian corpuscles is not always decided separately by augmented blood volume and by vasoconstricting drugs; that is, noradrenaline causes sometimes increased impulses from the corpuscles and sometimes decreased ones. The obtained results give the evidence that the positional relation between the vessel and the Pacinian corpuscle decides the two opposite response patterns of the corpuscles. However, it is likely that the increased distention of the ressels caused by augmented bloor volume sometimes gives effects on the positional relation different from those caused by drugs, and consequently they cause two opposite response patterns of corpuscles. On microscopic observation in the present experiments, the vessels constricted by the drug were dislocated more manifestly than the ones distended by an intra-venous injection of Krebs solution. And the frequency of afferent impulses decreased on hemorrhage, but the decrease was not always observed when acetylcholine was administered.

The results, in which any reflexes controlling the vascular system have failed to be found, make the possibility of 'vaso-controlling function of Pacinian corpuscles' unlikely, and their natural function is still unknown. Another possibility of their function is to control intestinal constriction and motility. Bergman ${ }^{11}$ asserted that in some person much accelerated stomach motility resulted in constriction of the stomach vessels under the hypersensitivity of vagus nerve, and it often caused ischemia and ulcer in the stomach. If Pacinian corpuscles really contribute to regulating the intestinal motility depending on the information about disordered abdominal blood pressure due to intestinal motility, the abdominal organs may be kept in good state.

Sorimachi et al. ${ }^{2}$ reported that adrenaline or bradykinin accelerated the function of isolated Pacinian corpuscles, and Selkurt ${ }^{13}$ observed that the occlusion of mesenteric artery maintained the blood pressure at a high level as long as 1 hour. This is too long to be regarded as a result of reflex action, but it is proper to presume hormonal effects. The role of chemical sensitivity of the Pacinian corpuscles in vascular regulation is also one of the problems to be studied hereafter.

\section{Acknowledgment}

We wish to express our thanks to Dr. W. R. Loewenstein for his guidance and advice.

\section{References}

1) Adrian, E.D. \& Umrath, K. The impulse discharge from the Pacinian corpuscle. J. Physiol. (Lond.), 1929, 68, 119-154.

2) Gray, J.A.B. \& Sato, M. Properties of the receptor potential in Pacinian corpuscles. I. Physiol. (Lond.), 1953, 122, 610-636.

3) Diamond, J., Gray, J.A.B. \& Sato, M. The site of initiation of impulses in Pacinian corpuscles. J. Physiol. (Lond.), 1956, 133, 54-67. 
4) Quilliam, T.A. \& Sato, M. The distribution of myelin on nerve fibres from Pacinian corpuscles. J. Physiol. (Lond.), 1955, 129, 167-176.

5) Loewenstein, W.R. On the specificity of a sensory receptor. J. Neurophysiol., 1961, 24, $150-158$.

6) Ishiko, N. \& Loewenstein, W.R. Effect of temperature on the generator and action potentials of a sense organ. J. gen. Physiol., 1961, 45, 105-124.

7) Ozeki, M. \& Sato, M. Intitiation of impulses at the non-myelinated nerve terminal in Pacinian corpuscles. J. Physiol. (Lond.), 1964, 170, 167-185.

8) Loewenstein, W.R. \& Mendelson, M. Components of receptor adaptation in a Pacinian corpuscle. J. Physiol. (Lond.), 1965, 177, 377-397.

9) Woollard, H.H. \& Weddell, G. The composition and distribution of vascular nerves in the extremities. $J$. Anat., 1935, 69, 165-176.

10) Gammon, G.D. \& Bronk, D.W. The discharge of impulses from Pacinian corpuscles in the mesentery and its relation to vascular changes. Amer. J. Physiol., 1935, 114, $77-84$.

1I) Bergmann, G. v. Katsch, G. Pathologische Physiologie spezieller Krankheitsbilder. Handbuch d. norm. u. path., Physiol., Berlin, J. Springer, Band 3, 1927, 1159-1198.

12) Sorimachi. M., Ban, N. \& Goto, K. Pacinian corpuscles and chemical agents. $J$. physiol. Soc. Jap. (Jap.), 1966, 28, 352.

13) Selkurt. E.E. Intestinal ischemic shock and the protective role of the liver. Amer. J. Physiol., 1959, 197, 281-285. 